\title{
Cómo son y cómo podrían ser las relaciones entre escuelas y familias en opinión del profesorado
}

\author{
Francisco-JuAN GARCÍA-BACETE \\ Universitat Jaume I, Castellón
}

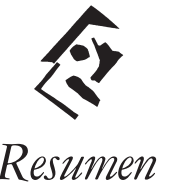

El objetivo de este estudio es conocer la opinión que tienen los profesores sobre las relaciones entre los centros escolares y las familias. En el estudio han participado 100 profesores de 38 centros escolares públicos de infantil y primaria de la provincia de Castellón, a los que se les aplicó un cuestionario.

De los resultados del estudio se desprende que la mayoría del profesorado se muestra satisfecho con la realidad actual de las relaciones entre escuelas y familias. Los profesores presentan el papel de los padres en la escolarización de los hijos como fundamental y como un deber, y aunque les atribuyen una diversidad de roles, en especial les reclaman que colaboren o contacten con ellos. Las formas de relacionarse más frecuentes son las que tienen al tutor como interlocutor. Los principales cambios propuestos son que las relaciones de las familias con los centros sean más frecuentes, más fluidas y respetuosas. Una quinta parte del profesorado propone una mayor apertura de los centros a las familias. Entre las dificultades identificadas figuran la falta de tiempo de los padres y la desconfianza hacia el maestro.

Palabras clave: Relaciones padres y profesores, formas, dificultades, propuestas.

\section{The nature of relations between school and family and what they could be like according to teachers}

\begin{abstract}
The study investigated teachers' opinions about relationships between the school and students' families. A representative sample of a bundred teachers from 38 infant and primary schools in the province of Castellon (Spain) who were asked to complete a questionnaire participated in the study. The results show that most teachers are satisfied with current relations between the school and families. Teachers consider that the role of parents in their child's schooling is fundamental and a duty; and although they attribute diverse roles to parents, they specially value their collaboration and personal contact. The most frequent types of relationships are those in which the child's school teacher acts as interlocutor. The main changes proposed by teachers are that relations between families and school should be more frequent, fluid, and respectful. A fifth of the teachers suggested that schools should be more open to families. The main difficulties identified are parent's lack of time and distrust towards the teacher.

Keywords: Parent-teacher relationships, types, difficulties, proposals.
\end{abstract}

Correspondencia con el autor: Francisco-Juan García-Bacete. Departamento de Psicología Evolutiva, Educativa, Social y Metodología. Universitat Jaume I. Avda Vicente Sos Baynat s/n 12071 Castellón de la Plana. E-mail: fgarcia@psi.uji.es

Original recibido: Julio, 2006. Aceptado: Octubre, 2006

(C) 2006 by Fundación Infancia y Aprendizaje, ISSN: $1135-6405$

Cultura y Educación, 2006, 18 (3-4), 247-265 
El profesor Vila en 1995, refiriéndose a las relaciones entre las escuelas y las familias, acuño la expresión "dos contextos y un solo niño". Esta expresión implica en primer lugar reconocer la unicidad del individuo que aprende, y al mismo tiempo, como bien ha descrito Trilla (2002), dos ideas complementarias: por una parte, que desde la perspectiva del sujeto que se educa es deseable que en todos los entornos educativos se tenga una visión holista, global de la educación del individuo y, por otra parte, que ningún entorno educativo educa "integralmente" a sus usuarios, entre otras cosas, por la obviedad de que si esto fuera posible sobrarían el resto de entornos educativos. Familias y escuelas tienen muchas razones para colaborar. García-Bacete (2003) destaca las siguientes: (1) los resultados de la investigación avalan ampliamente la importancia del hogar en los procesos educativos de los hijos (Christenson, Rounds y Gorney, 1992; Pérez, 2004). (2) porque los límites entre las experiencias de los niños en el hogar y las que experimentan en la escuela no están claros, tal y como han puesto de manifiesto los modelos ecológicos (concepto de mesosistema presente en el modelo de Bronfenbrenner 1979) y las corrientes constructivistas en educación (Coll, Martí y Mauri, 1993; Rodrigo y Arnay, 1997) (por ejemplo, el valor de los conocimientos y experiencias previas en los aprendizajes escolares, que unas veces tienen su origen en las aulas y otras, las más, en las calles y en los hogares). (3) En la literatura sobre escuelas eficaces se destaca el hecho de que las mejores escuelas, las que ofrecen un mayor apoyo a los padres y los niños obtienen logros superiores, siempre cuentan con padres que las apoyan y se encuentran integradas en sus barrios (Epstein, 1997; Marchesi, 2004). (4) La investigación (Henderson, 2002; Martínez-González, 1996) ha demostrado claramente que cuando los padres participan en la vida escolar no sólo se alcanzan un amplio número de efectos positivos, sino que los beneficiarios son diversos (el niño, los profesores, los padres, el centro escolar). Además, los constantes cambios sociales están produciendo una continua pérdida de recursos de familias y escuelas para hacer frente a sus funciones, lo que refuerza la necesidad de colaboración entre padres y profesores.

La relación entre los centros escolares y las familias puede concretarse en diversos tipos de actividades, desde las más institucionales, como la participación en los Consejos Escolares, a las más informales como pueden ser los intercambios de informaciones durante las entradas y salidas del colegio. Padres y profesores también pueden compartir la organización de actividades extraescolares, la participación en comisiones de convivencia o la difusión de las actividades de las escuelas de padres. En la mayoría de los centros se hacen reuniones con las madres y los padres, entrevistas orales y se elaboran informes escritos (GarcíaBacete, García, Villanueva y Doménech, 2003).

No obstante no siempre las relaciones que se producen entre profesores y padres son las deseables. Parte del problema, como señalan Terrón, Alfonso y Díez (1999) reside en que los canales de comunicación establecidos legalmente entre la familia y la escuela no son efectivos realmente y que la falta de un clima de confianza entre ambos sectores mina toda posible comunicación positiva y efectiva. Brynelsen (1984) señala un claro desequilibrio entre las demandas y respuestas que padres y profesores se intercambian entre sí. Solé (1996) destaca que los encuentros planificados son prácticamente inexistentes y cuando se dan es porque "toca" o "hay conflictos" e insiste en la necesidad de buscar iniciativas que tiendan al hecho de que las madres y los padres entren en la escuela y realicen un trabajo conjunto con los profesores, cada vez más sistemático. Finalmente, cabe destacar la falta de compromiso de la administración educativa, quien ha centrado todo su esfuerzo en las cuestiones de representatividad, y escasamente ha insistido en el funcionamiento y la formación de los distintos órganos e individuos. 
Fish (1990) clasifica las dificultades en las relaciones escuela-familia en cuatro grandes bloques: (1) posturas filosóficas -algunos profesores no están de acuerdo con la participación y algunos padres delegan completamente en la escuela-. (2) actitudinales -competitividad intergrupos, territorialidad y conflicto de valores-. (3) problemas logísticos -falta de tiempo, solicitar permisos laborales, tener que contratar el transporte o "canguros", familias monoparentales,...-. (4) habilidades de comunicación deficientes.

Las discrepancias señaladas adquieren mayor relevancia al tomar conciencia de que padres y profesores están obligados a encontrarse, que no pueden evitar relacionarse (García-Bacete, 2003). El reto educativo consiste en buscar la coordinación, asumiendo al mismo tiempo que las interferencias y conflictos no sólo son reales, sino hasta cierto grado inevitables, y que el supuesto de un medio educativo homogéneo y sin fricciones tampoco es deseable (Trilla, 2002). Nosotros pensamos que en la respuesta, las escuelas han de tomar la iniciativa, y aunque existen condicionantes fuera del control de los centros escolares, que el factor clave es la formación del profesorado en el trabajo con las familias. En primer lugar, la investigación ha mostrado importantes diferencias entre los profesores implicadores y los no implicadotes (Hulsebosch, 1991). Los primeros se refieren a los padres en términos positivos y como una fuente de recursos para los hijos y aceptan como valioso lo que el estudiante realiza fuera de la escuela, mientras que los no-implicadores se esfuerzan por mantener un control sobre la enseñanza, lo que les exige guardar la distancia necesaria con los padres. En segundo lugar, Conoley (1989) afirmó que podemos fracasar en nuestro trabajo si no conocemos a los padres de nuestros alumnos. Finalmente, tal y como afirman Dauber y Epstein (1993), la participación de las familias sólo se producirá si existe un esfuerzo deliberado por parte de la escuela.

Conviene, por tanto, saber lo que se hace actualmente en nuestros centros escolares escuchando las voces del profesorado.

\section{OBJETIVO}

La investigación tiene como objetivo principal conocer la opinión que tienen los profesores sobre las relaciones entre los centros escolares y las familias. Los objetivos específicos propuestos son conocer: a) ¿cuál es el papel de los padres en la escolarización de sus hijos?; b) ¿cómo son las relaciones entre las familias y las escuelas y viceversa?; c) si fuera el caso, ¿cómo piensan que deberían ser? y d) ¿cuáles son las principales barreras que dificultan el establecimiento de relaciones sistemáticas entre profesores y padres?

\section{MUESTRA}

La muestra está formada por 100 profesores de educación infantil y primaria de la provincia de Castellón. Los 100 profesores, 76 profesoras y 23 profesores, con un rango de edad entre 24 y 60 años, ejercen su actividad en 38 escuelas públicas de 19 localidades (68\% con más de 10.000 habitantes). La distribución por tamaño de los centros es representativa de esta provincia. El 66\% del profesorado imparte clase en educación primaria y el $34 \%$ en educación infantil. La muestra de profesores tiene una gran experiencia y antigüedad en el centro actual. El nivel sociocultural de las familias es fundamentalmente medio y medio-bajo (71\%).

\section{INSTRUMENTO}

Se ha utilizado un cuestionario formado por 125 preguntas (65 abiertas y 60 cerradas), distribuidas en 4 apartados generales (Plan de Acción Tutorial, El 
Tutor, Relaciones Escuela-Familia, Reuniones de Tutoría con los Padres, que se subdivide en 7 grandes apartados). En el presente trabajo sólo ofrecemos los resultados correspondientes a 7 preguntas incluidas en el apartado "Relaciones Escuela-Familia".

- En su opinión, ¿cuál es el papel de los padres en la escolarización de sus hijos?

- ¿De qué forma se relacionan las familias con su escuela?

Si corresponde, ¿cómo cree que debería ser la relación de las familias con la escuela? (intente concretar)

- ¿Cómo calificaría las relaciones de su escuela con las familias?

En concreto, ¿cómo se relaciona su escuela con las familias?

Si corresponde, ¿de qué forma debería relacionarse la escuela con las familias? (intente concretar)

- En su opinión, ¿cuáles son las principales dificultades/barreras para establecer (mejorar) unas relacionas sistemáticas y de colaboración entre las escuelas y las familias?

\section{VARIABLES}

Ubicación del Centro Escolar. Se han establecido dos niveles: a) Rural: Localidades con menos de 10.000 habitantes; b) Urbano: localidades con más de 10.000 habitantes.

Tamaño del Centro Escolar. Numero de Aulas. Se han seleccionado tres niveles: Pequeño (entre 7 y 9); Mediano (entre 10 y 18); Grande (más de 18).

Nivel Sociocultural de las Familias. Se solicitó a los profesores que indicaran el nivel sociocultural de los familias de los alumnos que asisten al colegio: B) Bajo/bajo medio, M) Medio y A) medio-alto/Alto.

Género del profesorado. Hombre, Mujer.

Edad del profesorado. Numero de años. Se han establecido 4 niveles de edad: 1) hasta 30 años, 2) entre 31 y 40 años, 3) entre 41 y 50 años, y 4 ) más de 50 años.

Ciclo educativo en el ejerce su labor docente. Se han realizado los siguientes agrupamientos: Etapa educativa (Infantil vs Primaria) y Edad de los Alumnos (Pequeños: infantil $+1^{\circ}$ ciclo de primaria; Grandes: $2^{\circ}$ y $3^{\circ}$ ciclo de primaria).

Experiencia del profesorado. Años de experiencia como profesor. Tres niveles: Jóvenes (entre 1 y 4); Medios (entre 5 y 9), Experimentados (10 ó más).

Antigüedad en un mismo centro. Años de permanencia en el centro escolar actual. Tres niveles: Noveles (primer año); Medios (entre 2 y 5); Antiguos (más de 5).

Titulación académica. Recoge el grado de formación académica del profesorado. Se han realizado dos tipos de agrupamientos: a) Maestro vs profesor con Otros estudios y b) Maestro vs. Licenciado.

\section{ANÁLISIS REALIZADOS}

Hemos realizado tres tipos de análisis:

(A) Se ha comenzado por un proceso de categorización de las respuestas a cada una de las preguntas.

(B) Se ha realizado un análisis de frecuencias y porcentajes de las categorías y del profesorado que las aporta.

(C) Por último, hemos realizado Análisis de Varianza de una vía (ANOVA) en función de factores de centro (localización, tamaño, nivel sociocultural de las familias) y del profesorado (género, edad, ciclo educativo, experiencia, antigüedad, titulación académica). 


\section{Proceso de Categorización}

Para poder analizar las respuestas hemos procedido en primer lugar a categorizar las contestaciones que ha dado el profesorado en cada una de las preguntas abiertas. Para ello hemos adaptado el procedimiento basado en la "grounded theory" por Carrero (en García-Bacete et al., 2003).

\section{Papel de los padres}

1) Fundamental. El rol de los padres es considerado como determinante, básico, fundamental, muy importante.

2) Responsabilidad. El proceso de escolarización de los hijos es una obligación y un deber de los padres. los padres son los primeros educadores, eligen centro, se aseguran que los hijos asistan al centro y que adquieran ciertos hábitos.

3) Centrados en el hijo. Se expresa el papel de los padres respecto de sus propios hijos: desde un genérico estar atentos a todo lo que afecta al hijo (interesarse) hasta participar directamente en la realización de sus tareas (apoyo).

4) Centrados en el centro y los profesores. Las respuestas expresan la relación de los padres con la escuela o con un profesor. subcategorías: (a) estar en contacto, (b) colaboración con el profesorado; (c) participación en la escuela; (d) participación en la ampa.

5) Quejas. Los profesores se quejan de la actuación de los padres.

\section{Relaciones familia-escuela y viceversa}

Con independencia de que la pregunta fuera cómo son las relaciones de las familias con su centro o como se relaciona su escuela con las familias, las categorías emergentes han sido las mismas, por lo que las presentamos a la vez. Sin embargo, no ha ocurrido lo mismo cuando nos hemos interesado por como deberían ser.

b1) ¿Cómo son las relaciones familia-escuela (y viceversa)

1) Presencial. Se requiere

1.1. Tutor. Las relaciones tienen como interlocutor o mediador la figura del tutor. Existen dos grandes conjuntos de respuestas en función del interés o de quienes participen:

1.1.1. Individual. Son encuentros básicamente formales entre el tutor y los padres de un alumno (la hora de tutoría, visitas de los padres, entrevistas personales, reuniones individuales, individualmente con,..).también se señalan las relaciones de carácter informal, o se alude a su frecuencia o forma (directa, continua, habitual, puntual)

1.1.2. Grupal. Se trata de reuniones del tutor con los padres de los alumnos de su grupo. Se utilizan diferentes acepciones para acompañar a la palabra reuniones (con el tutor, de tutoría, de padres, generales, de grupo, colectivas, trimestrales, periódicas)

1.2. Participación en actividades extraescolares. El motivo es la celebración de eventos especiales (extraescolares, fiestas, meriendas, carnavales, semana cultural, final de curso). No se suele especificar la forma de participar.

1.3. Organizacional. Se recoge el carácter institucional o de representación de las relaciones. Pueden ser a través: (a) de la dirección, (b) de la ampa o (c) del consejo escolar.

2) Otros medios

2.1. Escrita. Se trata básicamente de información escrita, habitualmente de carácter general. Los términos más usados son circulares, notas, boletines, informes. 
2.2. Otros medios. Son el teléfono y el correo.

b2) ¿Cómo deberían ser las relaciones de las familias con la escuela?

1) Incrementarse. El contenido común es pensar que las relaciones familiaescuela debería aumentar, en número, continuidad, intensidad o variedad.

2) Más fluida y respetuosa. El profesorado desea que la comunicación sea más abierta, y fluida y que los padres confíen en su tarea, les respeten y reconozcan su trabajo.

3) De colaboración. Se presenta un amplio abanico de colaboración, desde un continuar la labor iniciada por los docentes hasta un llegar a acuerdos.

4) Implicación de los padres. Una reivindicación constante de los docentes es que los padres se interesen por lo que hace su hijo.

5) Otras

b3) ¿Cómo deberían ser las relaciones de su escuela con las familias?

1) Conformismo. Se manifiesta satisfacción en su forma actual (está bien como está) o se repiten las respuestas dadas a preguntas anteriores (reuniones, entrevistas, tutorías).

2) Cambio. Mayor apertura y disponibilidad del centro hacia las familias y mayor, presencia y colaboración de las familias con el centro

\section{Dificultades en las relaciones entre las escuelas y las familias}

1) Falta de tiempo. El argumento principal es la falta de disponibilidad de los padres no pueden o no están disponibles, bien porque están muy ocupados o porque sus horarios no se lo permiten.

2) Familias situación de riesgo. Se apela a las características deficitarias de las familias: el nivel económico y sociocultural de los padres y/o del barrio y problemáticas familiares y sociales.

3) Falta de interés de los padres. Los padres no muestran interés por sus hijos, las tareas escolares o la escuela. Falta de motivación.

4) Desconfianza. Se refiere a los problemas de comunicación en general, pero sobretodo a la desconfianza de los padres hacia los maestros (dan la razón al hijo, critican al profesor,...) Y de éstos hacia las familias (se sienten controlados y censurados).

5) Desacuerdo roles. Padres y docentes muestran una percepción diferente de sus roles y funciones. Se habla de dificultad para establecer límites, usurpación de roles, infravaloración del docente y/o de la escuela junto a una delegación absoluta. El desacuerdo es presentado como una visión errónea de las familias.

6) Falta de implicación. La falta de tradición de familias y de escuela en favorecer la participación. La falta de participación, de implicación, de integración en el proceso educativo. También las estructuras de la escuela y modos de relación ya aprendidos.

7) Otras.

\section{Frecuencias}

Papel de los padres

Un porcentaje muy alto de profesores han contestado este ítem (94\%), aportando respuestas que hacen referencia a papeles diversos de los padres en la educación de los hijos. Los profesores lo ven claro: los padres sobretodo deben responder a las demandas de la escuela y colaborar con los profesores. La categoría "Centrado en el Centro" (las respuestas aportadas por los profesores o Enunciados (E) fue del $41.79 \%$, el porcentaje de Profesores (P) que aportan algún enunciado 
de esta categoría fue del $52.12 \%$ ) no sólo es la más frecuente, sino que se diferencia significativamente del resto de categorías $(p \leq .000)$, que quedan ordenadas de la siguiente forma:

TABLA I

Papel de los padres en la educación de los hijos $(E=189, P=94)$

\begin{tabular}{|c|c|c|c|c|c|c|c|c|c|c|c|c|}
\hline & 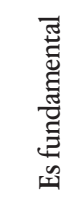 & 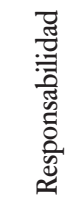 & 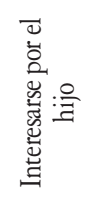 & 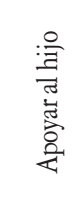 & 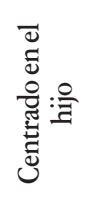 & 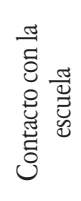 & 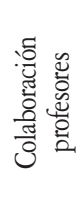 & 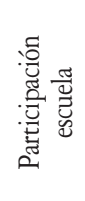 & 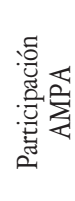 & 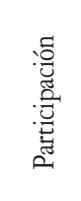 & 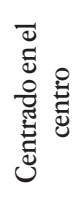 & $\frac{\frac{\pi}{\pi}}{\tilde{J}}$ \\
\hline $\begin{array}{c}\mathrm{N}^{\circ} \\
\text { Profesores }\end{array}$ & 27 & 28 & 19 & 14 & 29 & 15 & 33 & 14 & 4 & 15 & 49 & 7 \\
\hline $\begin{array}{c}\% \\
\text { profesores }\end{array}$ & 28.72 & 29.77 & 20.21 & 14.89 & 30.85 & 15.96 & 35.01 & 14.89 & 4.25 & 15.96 & 52.12 & 7.45 \\
\hline $\begin{array}{c}\mathrm{N}^{\circ} \\
\text { Enunciados }\end{array}$ & 31 & 31 & 21 & 17 & 38 & 19 & 39 & 15 & 6 & 21 & 79 & 10 \\
\hline $\begin{array}{c}\% \\
\text { Enunciados }\end{array}$ & 16.40 & 16.40 & 11.11 & 8.99 & 20.10 & 10.05 & 20.63 & 7.94 & 3.17 & 11.11 & 41.79 & 5,29 \\
\hline $\begin{array}{c}\% \\
\text { Enunciados clase }\end{array}$ & & & 55.26 & 44.74 & 100 & 24.05 & 49.36 & 18.98 & 7.59 & 26.58 & 100 & \\
\hline
\end{tabular}

El segundo foco de atención de los padres son los propios hijos $(\mathrm{E}=20.10 \%$, $\mathrm{P}=30.85 \%)$. Los profesores consideran que el papel de los padres respecto de los hijos recae en tareas como interesarse y estar atentos a todo lo que afecta al hijo (E $=11.11 \%, \mathrm{P}=20.21 \%)$ y apoyarle y ayudarle en la realización de sus tareas $(\mathrm{E}=$ $8.99 \%, \mathrm{P}=14.89 \%)$.

A continuación aparecen dos categorías de corte generalista con porcentajes de profesorado superior al $25 \%$ cada una. Los padres tienen un papel muy importante, fundamental en la escolarización de sus hijos $(\mathrm{E}=16.40 \%, \mathrm{P}=$ $28.72 \%)$, que no debiera olvidar en tanto que es su responsabilidad (E = $16.40 \%, \mathrm{P}=29.77 \%$ ). De aquí, pudiera derivar la presencia de la categoría "Quejas" ( $\mathrm{E}=5.29 \%, \mathrm{P}=7.45 \%)$, que aunque minoritaria, recoge el malestar de los profesores cuando se reduce al centro a "guardería de hijos".

\section{Satisfacción con las relaciones escuela-familia}

Un 60\% del profesorado califica las relaciones escuela-familia como óptimas (muy buena, muy positiva) y positivas (buena, positiva, satisfactoria, notable) frente a un $25 \%$ que las perciben como negativas o mejorables (insuficientes, escasa, puede ser mejor) y pésimas (desagradables, tensas, malas, imposibles). El resto de profesores utilizan adjetivos como normales, regulares, suficiente.

\section{Realidad}

Las relaciones presenciales son las más frecuentes, el 99\% de profesores dice que se dan estas relaciones tanto en el caso de las familias como en el de las escuelas. Por otra parte, mientras que un $28 \%$ del profesorado, además, usa otros medios (escrito, teléfono, correo), en el caso de las familias el porcentaje disminuye a un $10 \%$, refiriéndose exclusivamente a escritos. 
254 Culturay Educación, 2006, 18 (3-4), pp. 247-265

TABLA II

¿Cómo calificaría las relaciones de la escuela con las familias? $(E=120, P=97)$

\begin{tabular}{ccccccc}
\hline & Optima & Positiva & Media & Negativa & Pésima & Otras \\
\hline $\begin{array}{c}\mathrm{N}^{\circ} \\
\text { Profesores } \\
\%\end{array}$ & 8 & 50 & 26 & 19 & 6 & 2 \\
$\begin{array}{c}\text { Profesores } \\
\mathrm{N}^{\circ}\end{array}$ & 8.25 & 51.55 & 26.81 & 19.57 & 6.19 & 2.06 \\
$\begin{array}{c}\text { Enunciados } \\
\%\end{array}$ & 8 & 53 & 30 & 20 & 7 & 2 \\
Enunciados & 6.67 & 44.17 & 25 & 16.67 & 5.84 & 1.67 \\
\hline
\end{tabular}

El tutor es el vínculo más importante de las relaciones entre la escuela y las familias y de las familias con la escuela $(\mathrm{E}=49 \%, \mathrm{P}=84 \%, \mathrm{E}=61 \%, \mathrm{P}=94 \%$, respectivamente). En esta relación con el tutor como principal interlocutor, parece que las familias buscan preferentemente un encuentro individual $(\mathrm{E}=38 \%, \mathrm{P}$ $=81 \%)$ frente a los grupales $(\mathrm{E}=22 \%, \mathrm{P}=54 \%)$. Por su parte, desde la escuela las relaciones individuales y grupales aparecen equilibradas $(\mathrm{E}=26 \%, \mathrm{P}=64 \%$, $\mathrm{E}=23 \%, \mathrm{P}=58 \%$, respectivamente).

Dentro de las relaciones presenciales, las de carácter organizacional o institucional ocupan el segundo lugar, tanto en las relaciones de las familias con la escuela $(\mathrm{E}=21 \%, \mathrm{P}=33 \%)$ como de la escuela con las familias $(\mathrm{E}=21 \%, \mathrm{P}=$ $39 \%$ ). Dentro de esta categoría las relaciones a través del AMPA suponen más del $50 \%$ de las respuestas, seguidas de las que tienen lugar a través del Consejo Escolar y de las relaciones en las que participa algún miembro del equipo directivo, éstas últimas con porcentajes inferiores al $5 \%$.

TABLA III

¿Cómo se relacionan las familias con los centros escolares? $(E=229, P=92)$

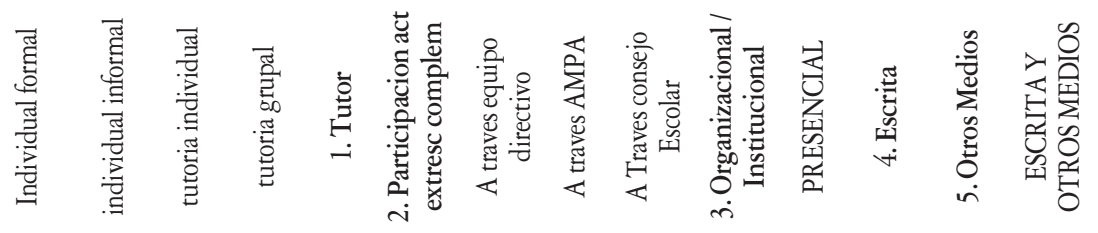

\begin{tabular}{|c|c|c|c|c|c|c|c|c|c|c|c|c|c|c|}
\hline $\begin{array}{c}\mathrm{N}^{\circ} \\
\text { Profesores }\end{array}$ & 58 & 22 & 74 & 50 & 86 & 22 & 5 & 26 & 16 & 30 & 91 & 9 & 1 & 9 \\
\hline $\begin{array}{c}\% \\
\text { Profesores }\end{array}$ & 63.0 & 23.9 & 80.4 & 54.4 & 93.5 & 23.9 & 5.4 & 28.3 & 17.4 & 32.6 & 98.9 & 9.8 & 1.1 & 9.8 \\
\hline $\begin{array}{c}\mathrm{N}^{\mathrm{o}} \\
\text { Enunciados }\end{array}$ & 64 & 24 & 88 & 51 & 139 & 25 & 6 & 26 & 17 & 49 & 213 & 14 & 1 & 15 \\
\hline $\begin{array}{c}\% \\
\text { Enunciados }\end{array}$ & 28 & 10.4 & 38.4 & 22.3 & 60.7 & 10.9 & 2,62 & 11.4 & 7.42 & 21.4 & 93 & 6.1 & 0.44 & 6.6 \\
\hline $\begin{array}{c}\% \\
\text { Enunciados clase }\end{array}$ & 46 & 17 & 63 & 37 & 100 & 100 & 12.2 & 53.1 & 34.7 & 100 & 100 & 93 & 6.67 & 100 \\
\hline
\end{tabular}

Por último, aproximadamente un cuarto del profesorado se refiere a la participación de los padres en las actividades extraescolares; más bien se limita a nombrar distintos eventos en los que los padres, en mayor o menor grado, suelen estar presentes. 
TABLA IV

¿Cómo se relaciona su centro escolar con las familias? $(E=177, P=67)$

\begin{tabular}{|c|c|c|c|c|c|c|c|c|c|c|c|c|c|}
\hline & 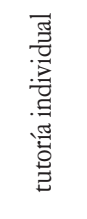 & 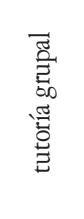 & ت. & 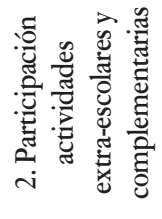 & 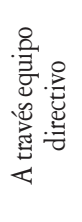 & 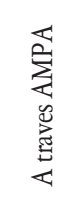 & 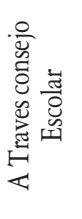 & 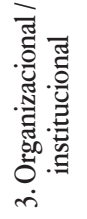 & 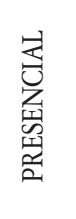 & 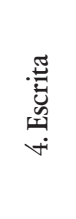 & 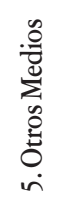 & 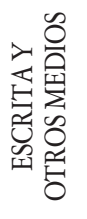 & 芯 \\
\hline $\begin{array}{c}\mathrm{N}^{\circ} \\
\text { Profesores }\end{array}$ & 43 & 39 & 56 & 19 & 6 & 19 & 11 & 26 & 66 & 17 & 6 & 19 & 8 \\
\hline $\begin{array}{c}\% \\
\text { Profesores }\end{array}$ & 64.18 & 58.21 & 83.58 & 28.36 & 8.97 & 28.36 & 16.42 & 38.81 & 98.51 & 25.38 & 8.96 & 28.36 & 11.94 \\
\hline $\begin{array}{c}\mathrm{N}^{\circ} \\
\text { Enunciados }\end{array}$ & 46 & 40 & 86 & 25 & 7 & 19 & 12 & 38 & 149 & 21 & 7 & 28 & 9 \\
\hline $\begin{array}{c}\% \\
\text { Enunciados }\end{array}$ & 25.99 & 22.60 & 48.59 & 14.97 & 3.95 & 10.74 & 6.78 & 21.47 & 84.18 & 11.86 & 3.95 & 15.82 & 5.09 \\
\hline $\begin{array}{c}\% \text { e } \\
\text { Enunciados clas }\end{array}$ & 53.49 & 46.51 & 100 & 100 & 18.42 & 50.00 & 31.58 & 100 & & 75.00 & 25.00 & 100 & \\
\hline
\end{tabular}

\section{Cambio}

Los profesores formulan cuatro demandas a los padres: las tres primeras se centran en el modo en que debería desarrollarse la conexión entre la familia y la escuela, mientras que la cuarta pone a los padres en relación con su propio hijo.

En opinión del profesorado, las familias en primer lugar deberían relacionarse más con la escuela y adoptar formas más diversas $(\mathrm{E}=31.62 \%, \mathrm{P}=42.86 \%)$ y esforzarse por comunicarse de forma más abierta y cordial, mostrando confianza y reconocimiento del trabajo docente $(\mathrm{E}=34.18 \%, \mathrm{P}=42.86 \%)$. Entre estas dos categorías suman el $65.80 \%$ de los enunciados. A continuación, los profesores reclaman mayor colaboración de los padres, desde un simple continuar la tarea del maestro hasta adoptar un mayor compromiso $(\mathrm{E}=21.37 \%, \mathrm{P}=$ $28.57 \%$ ).

Por último, los profesores piensan que las familias no se interesan lo suficiente por la educación, por lo que hacen sus hijos en las escuelas. A los padres, dicen, les falta interés $(\mathrm{E}=7.69 \%, \mathrm{P}=11.43 \%)$.

TABLA V

¿Cómo deberían relacionarse las familias con los centros escolares? $(E=117, P=70)$

\begin{tabular}{|c|c|c|c|c|c|}
\hline & Incrementarse & $\begin{array}{c}\text { Comunicación } \\
\text { más fluida y } \\
\text { respetuosa }\end{array}$ & $\begin{array}{l}\text { Relaciones de } \\
\text { colaboración }\end{array}$ & $\begin{array}{l}\text { Implicación de } \\
\text { los padres }\end{array}$ & Otras \\
\hline $\begin{array}{c}\mathrm{N}^{\circ} \\
\text { Profesores }\end{array}$ & 30 & 30 & 20 & 8 & 5 \\
\hline $\begin{array}{c}\text { Profesores } \\
\% \\
\text { Profesores }\end{array}$ & 42.86 & 42.86 & 28.57 & 11.43 & 7.14 \\
\hline $\begin{array}{c}\mathrm{N}^{\mathrm{o}} \\
\text { Enunciados }\end{array}$ & 37 & 40 & 25 & 9 & 6 \\
\hline $\begin{array}{c}\% \\
\text { Enunciados }\end{array}$ & 31.62 & 34.18 & 21.37 & 7.69 & 5.13 \\
\hline
\end{tabular}


TABLA VI

¿Cómo debería relacionarse su centro con las familias? $(E=83, P=39)$

\begin{tabular}{cccccccc}
\hline & Actualidad & Lo que hago & Satisfecho & $\begin{array}{c}\text { Cambio } \\
\text { familia }\end{array}$ & $\begin{array}{c}\text { Cambio } \\
\text { escuela }\end{array}$ & Cambio & Otras \\
\hline $\begin{array}{c}\mathrm{N}^{\circ} \\
\text { Profesores } \\
\%\end{array}$ & 9 & 5 & 14 & 9 & 20 & 26 & 4 \\
$\begin{array}{c}\text { Profesores } \\
\mathrm{N}^{\circ}\end{array}$ & 23.06 & 12.82 & 35.90 & 23.08 & 51.29 & 66.67 & 10.26 \\
$\begin{array}{c}\text { Enunciados } \\
\%\end{array}$ & 9 & 13 & 22 & 18 & 38 & 56 & 5 \\
$\begin{array}{c}\text { Enunciados } \\
\%\end{array}$ & 10.84 & 15.66 & 26.51 & 21.69 & 45.78 & 65.12 & 6.02 \\
Enunciados clase & 40.90 & 59.09 & 100 & 32.14 & 67.86 & 100 & \\
\hline
\end{tabular}

Preguntados los profesores sobre cómo deberían relacionarse con las familias, los profesores en primer lugar dirimen si hay necesidad de cambios o no, y en el caso de que lo sean, a quien le corresponden. Así, un 35.90\% del profesorado muestra satisfacción con lo que las escuelas hacen. Quizás, por ello, sólo 39 profesores han contestado a esta pregunta.

Las otras dos terceras partes del profesorado indican cambios necesarios, si bien un tercio de ellos se aprestan a nombrar, de nuevo, cambios que deberían hacer las familias (que vengan al centro, que se impliquen más,..). Finalmente, en torno a la mitad de profesores y enunciados $(\mathrm{P}=51.29 \%, \mathrm{E}=45.78 \%)$ se refieren a cambios en la escuela, apuntando en general una mayor apertura de las escuelas. Los profesores deberían mostrar mayor disponibilidad y aceptación de las opiniones de los padres, un mayor uso de las infraestructuras de la escuela en horario no escolar, horarios más amplios para atender a los padres.

\section{Dificultades}

Los profesores han identificado 6 barreras para que las relaciones escuelafamilia sean las deseables. La falta de tiempo, en especial de los padres, es la principal dificultad. Casi la mitad de los profesores $(\mathrm{P}=43.48 \%)$ han dado alguna respuesta en este sentido: los padres están muy ocupados, no disponen de tiempo para dedicarlo a los hijos o a la escuela, sus horarios laborales no se lo permiten o existe incompatibilidad con los horarios que las escuelas programan para reunirse con los padres.

Las dificultades de comunicación entre padres y profesores constituyen la segunda gran barrera $(\mathrm{E}=18.47 \%, \mathrm{P}=27.17 \%)$. Parece que ni unos ni otros tienen las habilidades necesarias para hacerse entender o para ponerse en lugar del otro. Padres y profesores no confían entre sí, mayoritariamente en el caso de los padres hacia los maestros. En opinión de los profesores, los padres se ponen a la defensiva ante los comentarios que el maestro hace de sus hijos, les justifican y no están dispuestos ni a ver sus déficits ni a entender que no siempre pueden ser los mejores. Un pequeño porcentaje de respuestas $(\mathrm{E}=3.19 \%)$ también muestran que los docentes recelan de los padres, se sienten presionados, piden que les dejen trabajar.

Las dos categorías siguientes aluden a las características de las familias. Los hijos, la educación de los hijos o la vida escolar de sus hijos no están entre las principales motivaciones de los padres. A los padres les falta interés, dicen el $20.65 \%$ de los profesores $(E=13.38 \%)$. Las condiciones de vida o los problemas 
Como son y como podrían ser las relaciones entre escuelas y familias / F.-J. García-Bacete

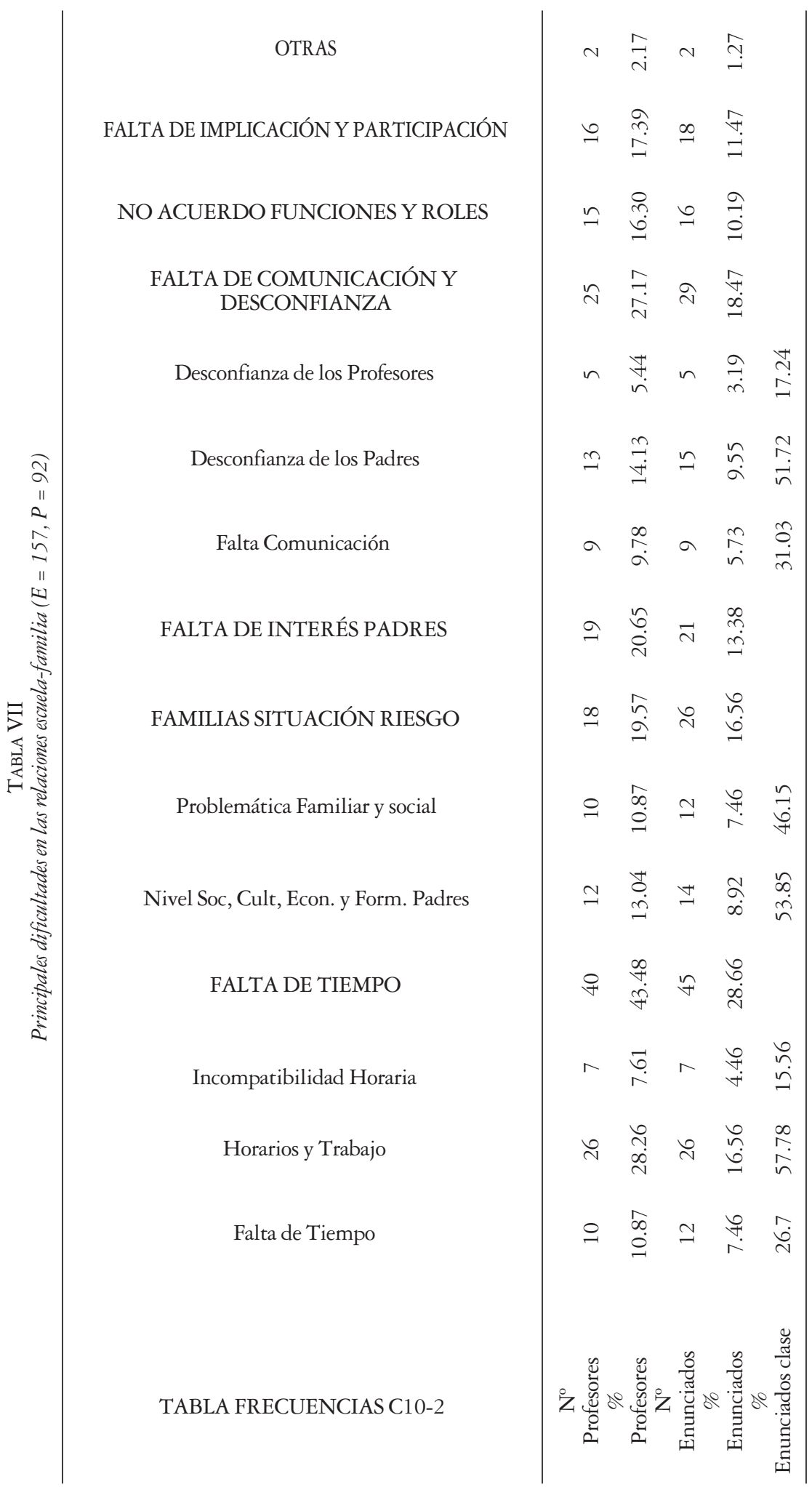


en la familia también son vistos como una fuente importante de dificultades (E $=16.36 \%, \mathrm{P}=19.57 \%$ ). Los profesores se refieren a ellos con expresiones como el nivel cultural, la condición social, la falta de información de algunas familias, la falta de comunicación dentro de la familia o familias desestructuradas.

Finalmente, encontramos dos categorías directamente vinculadas con la relación entre la familia y la escuela. En el plano de las concepciones, la categoría "desacuerdo de roles" ( $\mathrm{E}=10.19 \%, \mathrm{P}=16.30 \%)$, expresa que unos y otros tienen diferentes ideas sobre lo que es la escuela y de los roles que cabe esperar de padres y docentes. Los límites entre los derechos y deberes, los roles de unos y otros no están claros o se viven como una usurpación. En un extremo los padres se desentienden y consideran que la educación es tarea de la escuela. En el plano de las acciones, en la categoría "falta de participación" $(\mathrm{E}=11.47 \%, \mathrm{P}=$ $17.39 \%$ ), se denuncia una falta de tradición por parte de familias y de escuela en favorecer la participación de los padres en la educación escolar de los hijo y, en consecuencia, los bajos niveles de participación de los padres.

\section{Análisis de Varianza (ANOVAS)}

Para cada uno de los procesos de categorización hemos realizado ANOVAs de un factor para cada una de las variables del centro y del profesor empleadas. Los resultados agrupados por pregunta se observan en las tablas VIII, IX, X y XI. Sólo se presentan los ANOVAS con niveles de significación $p \leq .05$.

\section{Papel de los padres}

Las diferencias significativas están asociadas a los tres factores de centro, especialmente localización y el tamaño del centro, y a sólo dos del profesorado (ciclo y titulación).

Los profesores de centros ubicados en zonas rurales, los de los colegios pequeños y los que ejercen en educación primaria son los que más enunciados aportan a la categoría centrado en el centro. Los colegios pequeños especifican la participación de los padres a través de la AMPA y los rurales la participación en actividades de la escuela. Por su parte, los profesores de colegios rurales, de colegios pequeños y los que dan clase a los ciclos medio y superior de primaria destacan el rol de los padres como colaboradores de los profesores.

El papel de los padres centrado en los hijos es destacado por profesores que trabajan en colegios urbanos y por los profesores que tienen alguna licenciatura; en concreto, se refieren al apoyo que los padres deberían dar a sus hijos. Por su parte, los profesores de colegios con familias de nivel educativo extremos, tanto bajo como alto, prefieren insistir en que los padres se interesen por sus hijos.

Finalmente, apelar a la responsabilidad de los padres es más frecuente entre los profesores de colegios localizados en zonas urbanas y de tamaño medio.

\section{Satisfacción}

Como ya comentamos los profesores se muestran mayoritariamente satisfechos con las relaciones entre escuelas y familias. No obstante, aunque pocos, si existen algunos resultados diferenciales. Los profesores de escuelas a las que asisten alumnos de familias con alto nivel sociocultural son los que más califican las relaciones entre la escuela y la familia como óptimas. Los profesores de centros rurales dicen que son positivas y los de medios urbanos las califican de normales. La visión más pesimista la tienen los profesores de colegios grandes, los que trabajan en infantil y los que tienen una antigüedad media en el colegio. 


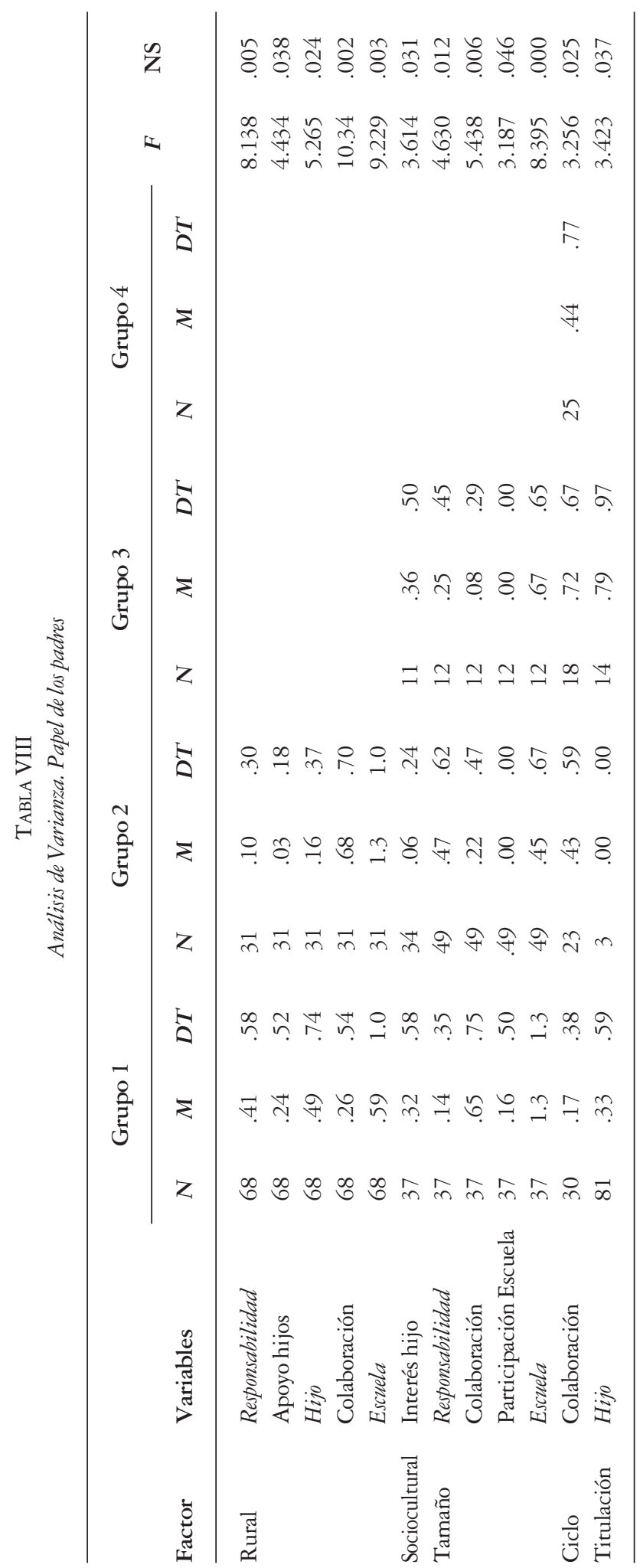


Realidad

El ciclo educativo en el que se imparte clase, el género del profesor y el nivel sociocultural de las familias son los factores que más efectos diferenciales aportan a las relaciones entre escuelas y familias. Estos tres factores acumulan 13 de las 16 diferencias significativas encontradas. Diferencias que están presentes sobretodo cuando se analiza las relaciones de las familias con el centro (13 diferencias).

La actividad del tutor en general es destacada por los profesores varones y los de infantil. El carácter colectivo de la tutoría es remarcado por las profesoras, los profesores más jóvenes y novatos y los que dan clase en infantil, mientras que en los colegios situados en contexto de nivel sociocultural alto se priorizan los encuentros de tipo individual formal.

Las relaciones de tipo institucional o de representación en su conjunto predominan en los colegios con familias de nivel sociocultural medio. Ahora bien cuando se trata de especificar con quien se establece esta relación encontramos algunas diferencias. Los profesores de infantil destacan las relaciones en el Consejo escolar, los profesores de niños más mayores y los profesores varones afirman que las relaciones más frecuentes son con el equipo directivo, y los profesores con otros estudios y los de colegios con familias con niveles socioculturales medios piensan que las familias se relacionan sobretodo a través de las AMPAs.

Las actividades extraescolares sólo han participado en una diferencia significativa: los profesores de las etapas educativas más altas (ciclo medio y superior) observan una mayor participación de los padres en actividades extraescolares que los profesores de otros niveles.

\section{Cambio}

Parece que existe un acuerdo bastante generalizado entre el profesorado respecto de cómo deberían ser las relaciones de las familias con las escuelas y de las escuelas con las familias. Tan sólo han aparecido cuatro efectos diferenciales: los profesores de centros urbanos desearían que las familias se comunicaran con la escuela de forma más abierta y cordial, que se confíe en su trabajo. De forma complementaria, los profesores de centros rurales son los que mayor conformidad expresan con las relaciones actuales entre padres y profesores. Por su parte, los profesores de colegios grandes y los que ejercen en el ciclo inicial piensan que las familias deberían relacionarse más con la escuela.

\section{Dificultades}

Al igual que en los apartados anteriores el número de diferencias significativas es pequeño. Pero en este caso en la mayoría de los factores aparece algún efecto diferencial.

Para los profesores de escuelas rurales el horario de trabajo de los padres es una dificultad mayor que en los centros urbanos. Las dificultades asociadas a las familias en situaciones de riesgo, en particular las derivadas de su nivel social y cultural, son menos visibles a medida que los centros se van haciendo más grandes.

La desconfianza y los problemas de comunicación entre padres y profesores es especialmente percibida por los maestros-licenciados. Los problemas de comunicación en general, sin que necesariamente conlleve recelos y desconfianzas entre padres y profesores, son aportados por profesores jóvenes, con poca experiencia y nuevos en el centro y por profesores que dan clase en centros a los que asisten niños cuyas familias tiene alto nivel sociocultural. 
Como son y como podrían ser las relaciones entre escuelas y familias / F.-J. García-Bacete

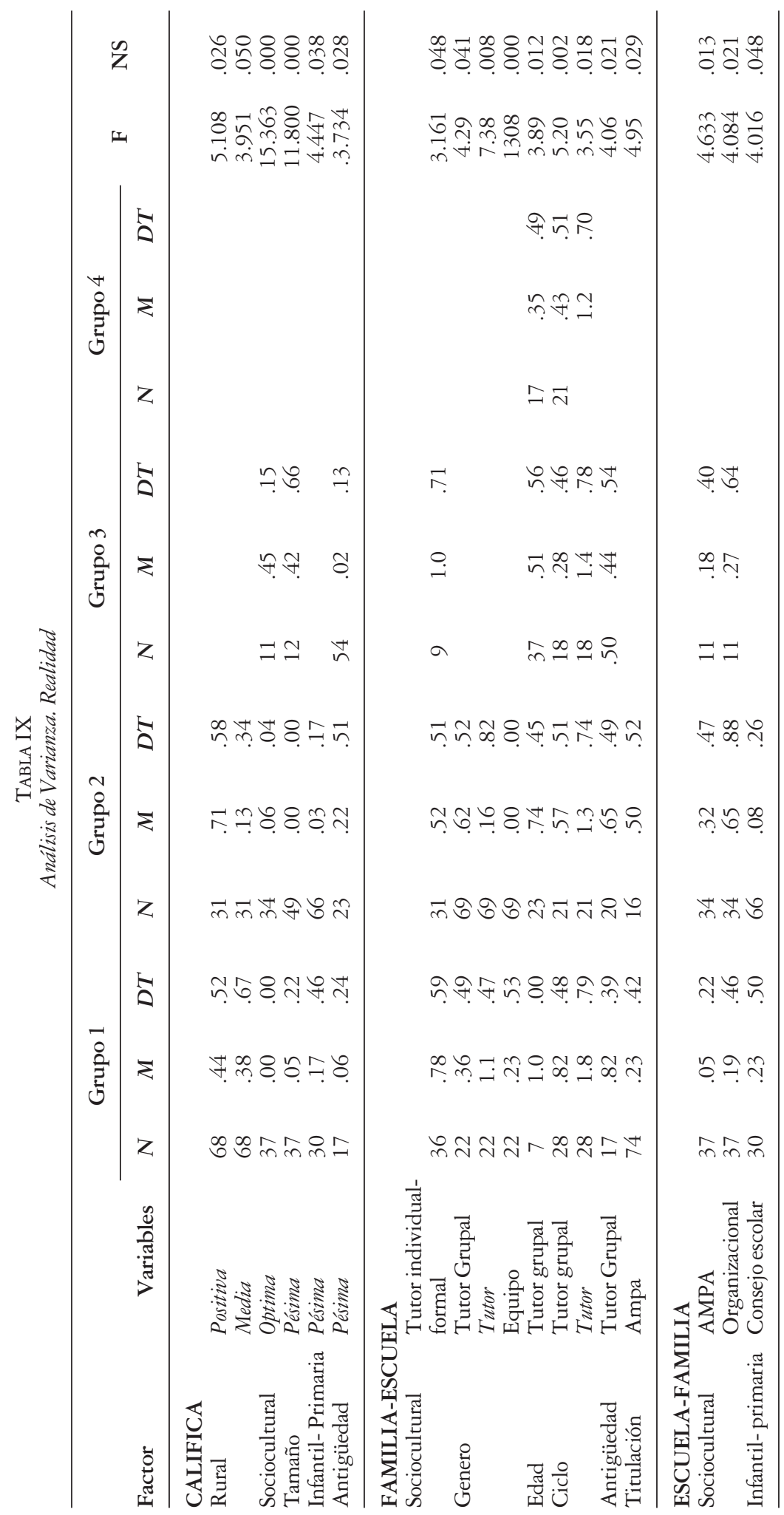




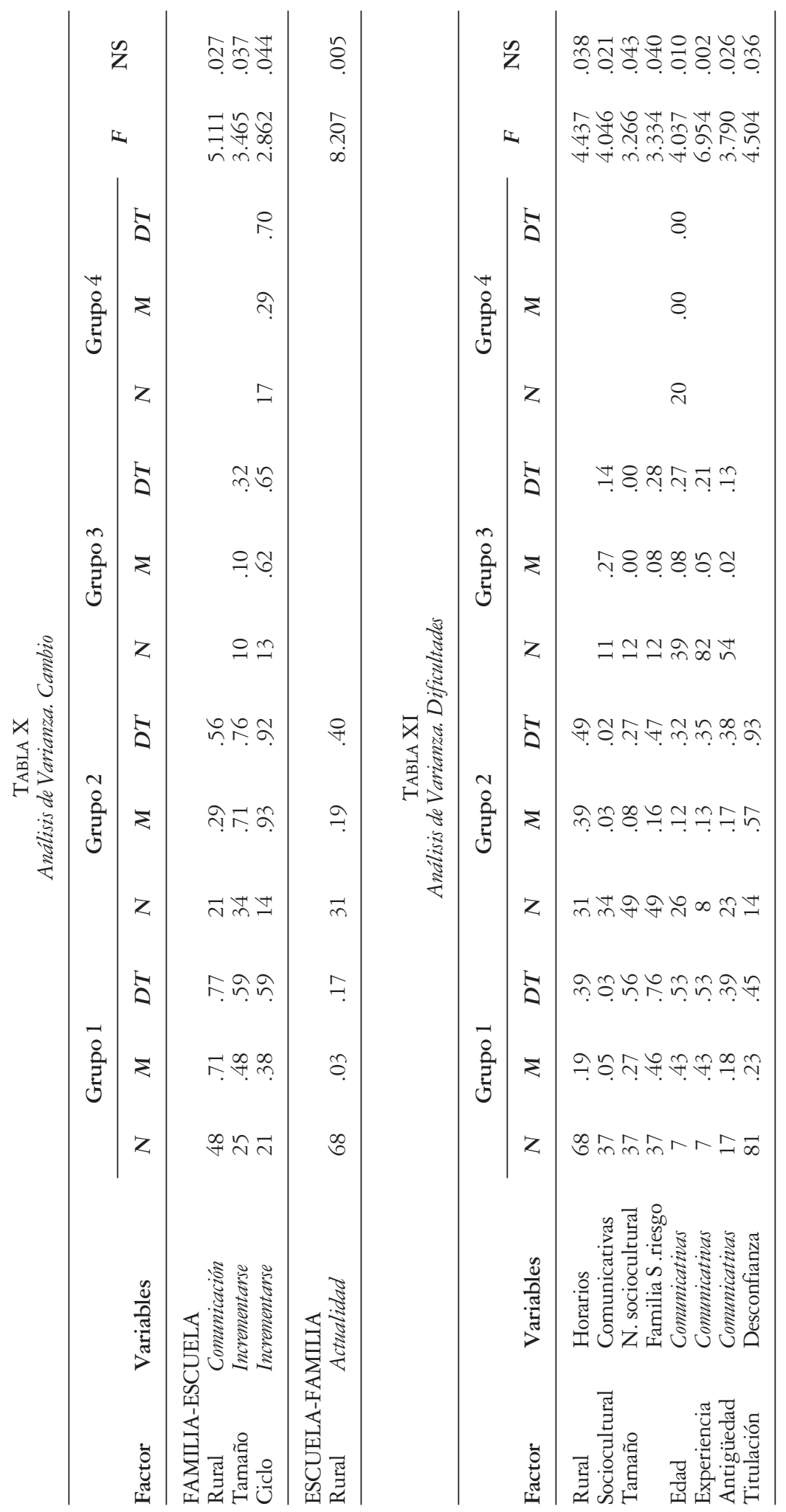




\section{DISCUSIÓN}

El profesorado se muestra mayoritariamente satisfecho con las relaciones actuales entre escuelas y familias y perciben el papel de los padres en la escolarización de los hijos como algo fundamental y una responsabilidad de los padres. Los profesores atribuyen una diversidad de roles a los padres respecto de la escuela y al propio hijo. La forma de relacionarse se refiere casi exclusivamente a la presencia de los padres en el centro escolar y de forma muy mayoritaria se realizan a través del tutor, en especial cuando se habla de las relaciones de las familias con los centros, quizás como reflejo de la legalidad. Otras formas frecuentes de relacionarse son las actividades extraescolares, una de las formas de participación mejor vista por los profesores, y las relaciones a través de las AMPAs.

Los principales cambios propuestos son que las relaciones de las familias con los centros sean más frecuentes, más fluidas y respetuosas y, en menor medida, se propone una mayor apertura de los centros a las familias. La falta de tiempo y de interés de los padres y la desconfianza hacia el maestro son las principales dificultades mencionadas.

Con respecto a los análisis de varianza, los tres factores de centro aportan más del $50 \%$ de los efectos diferenciales encontrados. Los profesores de centros rurales valoran como óptimas las relaciones con las familias, defienden un rol de los padres orientado hacia el centro e indican que los horarios de los padres son la principal dificultad. Por su parte, los de los colegios urbanos califican las relaciones con las familias sólo como normales y reclaman mayor cordialidad por parte de las familias. En su opinión, colaborar con los profesores, pero sobretodo centrarse en sus hijos, apoyarles, es una responsabilidad de los padres.

Para los profesores de colegios con familias con nivel sociocultural alto, a pesar de los problemas de comunicación que señalan, las relaciones con la familias son óptimas y afirman que los padres deberían centrarse en sus hijos, por ello postulan las relaciones formales con el tutor de interés individual. En los de nivel sociocultural medio se prefiere que los padres desarrollen relaciones institucionales, sobretodo a través de las AMPAs.

Los profesores de colegios grandes viven las relaciones con las familias como pésimas (también los de infantil y los que llevan un cierto tiempo en el colegio) y piensan que como primera medida deberían incrementarse. Por su parte, los de colegios medianos prefieren apelar a la responsabilidad de los padres. Los centros pequeños identifican los niveles económicos y culturales de los padres y la problemática familiar como importantes dificultades, quizás por ello defienden que el papel de los padres debería focalizarse en el centro, colaborando con el profesor y buscando ayuda o participando con la AMPA.

Varias cuestiones de interés pensamos que emergen en el estudio y que mantienen cierta relación entre sí. En primer lugar, destacar que los profesores son conscientes de la importancia de los padres en la escolarización de los hijos. En segundo lugar, mayoritariamente se sienten satisfechos en cómo están las cosas en la actualidad. En tercer lugar, informan que si fueran necesarios cambios estos corresponden mayoritariamente a los padres. Y en cuarto lugar que las dificultades principales en la actualidad también recaen en el ámbito de las familias.

En realidad, ¿cual es el papel que los profesores esperan de los padres?, ¿los resultados muestran satisfacción o conformismo? Y lo que es más interesante, ¿qué podemos esperar del profesorado, de los directores y de las escuelas?

Pensamos que lo que los profesores pueden estar señalando es una relación unidireccional, en la que se enfatiza la "responsabilidad " de los padres de apoyar a la escuela y a los profesores para que éstos puedan cumplir con sus objetivos. Que la categoría “centrados en la escuela” sea la más frecuente, en particular la 


\section{Cultura y Educación, 2006, 18 (3-4), pp. 247-265}

subcategoría colaboración con el profesorado, la misma presencia de una categoría de "quejas", su insistencia en la responsabilidad de los padres y la casi ausencia de cambios o dificultades atribuibles a la escuela y al profesorado puede ayudar a entender esta afirmación.

¿Satisfechos o conformistas? Nosotros pensamos que cuando la mayoría de los análisis señalan una situación preocupante, y se habla de ausencia de relaciones cuando no de confrontación, los resultados encontrados están más cerca de expresar conformismo que satisfacción. Un 25\% del profesorado las valora como negativas o pésimas, el número de profesores que aportan enunciados a las preguntas del tipo "como deberían" desciende de forma notable, en especial en el caso de los cambios en la escuela (sólo 30 profesores) como diciendo "no más tareas por favor (no sin razón, ya que la sobrecarga de trabajo es notoria en el profesorado), además, cuando se les pregunta por los cambios más de un tercio contesta con las bondades del sistema actual, que no quiere decir que ignoran las dificultades existentes ( 92 profesores mencionan al menos una), y que el $76 \%$ de los propuestos afectan a las familias.

¿Qué podemos esperar de los profesores, de los directores y de la escuela?. Si analizamos los cambios propuestos se observa que se trata de una respuesta lógica a las dificultades (ver entre paréntesis). Se apunta que las familias deberían incrementar sus relaciones con la escuela (falta de tiempo, familias en situación de riesgo), que éstas deberían ser más fluidas y respetuosas (desconfianza, desacuerdo), implicarse más en los propios hijos (falta de interés) y colaboración con la escuela y los profesores (falta de tradición). Lo que a su vez, guarda cierto paralelismo con lo propuesto por Fish (1990): problemas logísticos, falta de habilidades comunicativas, dificultades actitudinales y dificultades de carácter epistemológico. Ni que decir tiene que Fish hablaba de dificultades atribuibles tanto a padres como a profesores.

De esta comparación surge una cierta contradicción. Los cambios que se le piden a la familia en parte son atribuibles a las dificultades existentes, pero éstas, en gran parte, están fuera del control de las familias. La mayor parte de los enunciados reflejan las dificultades de los padres para conciliar la vida familiar, y en particular su implicación con los centros escolares, con la vida laboral. Dos tercios de los enunciados hablan de familias en situación de riesgo, de horarios laborales e incompatibilidad horaria, de que la educación o los hijos no están entre las principales motivaciones de los padres, quizás como consecuencia de los dos primeros ítems.

Señalar a los padres como los principales responsables de la situación actual y no confiar en su iniciativa o propuestas significa menospreciar el capital social que aportan los padres a la relación (Symeous, 2006). En muchas ocasiones cuando los profesores se quejan de la poca participación de los padres no se suele tener en cuenta la diversidad que existe entre las familias y sus propias dificultades (Marchesi, 2004). Se suele pensar únicamente en cómo los padres han de colaborar con la escuela, olvidando, por lo general, en cómo la escuela debería favorecer la colaboración de y con las familias (García-Bacete, Górriz, Cloquell, 2004). Hace falta contemplar modelos en los que se tenga en cuenta las necesidades y recursos de los profesores como de los padres (Hornby, 1990).

Sin lugar a dudas, las interacciones participadas por el tutor han sido las más numerosas. Quizás como reflejo de lo que dice la legislación. Lamentablemente, nada se dice de la formación de los tutores ni de las condiciones que hagan posible una comunicación positiva y efectiva entre profesores y padres. Un alto porcentaje de tutores afirma que no han recibido ninguna formación, que la tutoría exige del maestro ser un experto en relaciones interpersonales y tener una dedicación absoluta, por lo que no extraña que identifiquen las relaciones con los 
padres y la falta de tiempo como sus principales dificultades (García-Bacete, García y Doménech, 2005). Por otra parte, al concentrarnos en la figura del tutor, por una parte nos alejamos de los aspectos de contenido pedagógico del aula (Bassedas, Huguet y Solé, 1996) y, por otra parte, se olvida la importancia de una organización de los centros que potencia y hace posible dicha colaboración, y en particular del papel de la dirección. Nuestros datos revelan una ausencia de políticas de participación en las escuelas (no tradición, no cambios en las escuelas, focalización excesiva en el tutor, pobre presencia de los enunciados organizativos) y un ejercicio muy pobre por parte de la dirección en el cumplimiento de su función de fomentar la participación de los padres, o cuando menos es algo desconocido para el profesorado.

En definitiva, tenemos la obligación de hacer más atractiva la escuela (la educación, los hijos, los alumnos) para padres y profesores e iniciar acciones "posibles y cotidianas" en el marco de propuestas más globales y sistemáticas (GarcíaBacete, 2003).

\section{Referencias}

Bassedas, E., Huguet, T. \& Solé I. (1996). Aprendre i ensenyar a l'educació infantil. Barcelona: Graó.

BRonfenbrenner, U. (1979). The ecology of human development. Cambridge: Harvard University Press.

BRYNELSEN, D. (1984, Ed.). Working together. A handbook for parent and professionals. Ontario, Canada: National Institute. on Mental Retardation (disponible sólo en microficha).

Christenson, S. L., Rounds, T. \& GoRneY, D. (1992). Family factors and student achievement: An avenue to increase student's success. School Psychology Quarterly, 7, 178-206.

COLl, C., MARTí, E. \& MAURI, E. (Comps.) (1993). El constructivismo en el aula. Barcelona: Graó.

CONOLEY, J. C. (1989). The school psychologist as a community-family service provider. En R. C. D'Amato \& R. S. Dean (eds): The school psychologist in nontraditional settings (pp 33-65). Hillsdale, NJ: LEA.

DAUBER, S. L. \& EPSTEIN, J. L. (1993). Parent attitudes and practices of involvement in inner-city elementary and middle schools. En N. F. Chavkin (Ed.), Families and schools in a pluralistic society (pp. 205-216). Albany: State University of New York Press.

EPSTEIN, J. (1997). School, family and community partnership. Thousand Oaks, CA: Corwin Press, INC.

FISH, M. C. (1990). Best practices in family-school relationships. En A. Thomas y J. Grimes (eds): Best practices in school psychology-II (pp 383-392). Washington, DC: NASP.

GARCÍA-BACETE, F. J. (2003). Las relaciones escuela-familia: un reto educativo. Infancia y aprendizaje, 26 (4), 425-437.

García-Bacete, F. J., García, R., Villanueva, L. \& DoméneCh, F. (2003). Funciones y actividades que realizan los profesores tutores en los centros escolares de infantil y primaria de la provincia de Castellón. Revista Galego-Portuguesa de Psicoloxia e Educación 7 (9), 113-126.

GarCía-BACETE, F. J., Górriz, A. \& Cloquell, R. (2004). El papel de los padres en la escolarización de los hijos. El punto de vista del profesorado. Comunicación presentada al IV Congreso Internacional de Psicología y Educación. Almería: Universidad de Almería.

GarCía-BACEte, F. J., GarCíA, R. \& DomÉNECH, F. (2005). Características, dificultades y necesidades formativas de los profesores tutores de educación infantil y primaria en los centros escolares de la provincia de Castellón. Revista Española de Orientación y Psicopedagogía, 16 (2), 199-224.

Henderson, A. (2002). A New Wave of Evidence: The Impact of School, Family and Community Connections on Student Acbievement Washington, DC: National Committee for Citizens in Education.

HoRnBY, G. (1990). The organisation of parent involvement. School Organisation, 10 (2-3), 247-252.

Hulsebosch, P. L. (1991). Beauty the eye of the beholder: How and why teachers involve parents. International Journal of Educational Research, 15 (2), 183-200.

MARCHESI, A. (2004). Que será de nosotros los malos alumnos. Madrid: Alianza Editorial

Martínez-González, R. A. (1996). Familia y educación. Oviedo: Servicio de Publicaciones Universidad de Oviedo.

PéreZ, E. M.(2004). La influencia de variables familiares, personales y escolares en los resultados de los alumnos. Madrid: Universidad Complutense (Tesis Doctoral)

RodRIGO, M. J. \& ARNAY, J. (Comps.) (1997). La construcción del conocimiento escolar. Barcelona: Paidós.

SoLÉ, I. (1996). Las relaciones entre la familia y la escuela. Cultura y Educación, 4, 11-17.

SYMEOUS, L. (2006). Cultural and social capital: What can we learn for investigating and strengthening school-family collaboration? Cultura y Educación, Monográfico, diciembre

Terrón, E., AlFonso, C. \& Dízz, E. (1999). Construir la comunicación entre la familia y la escuela como una relación de confianza. Aula de Innovación Educativa, 85, 6-10.

TrILLA, J. (2002). La respuesta del marco escolar frente a las nuevas necesidades de la familia y el educador. Aula de Innovación Educativa, 112, 39-43.

VILA, I (1995). Familia y escuela: dos contextos y un solo niño. Aula de Innovación Educativa, 45, 72-76. 